\title{
Acceleration vs Accuracy: Influence of Basis Set Quality on the Mechanism and Dynamics Predicted by Ab Initio Molecular Dynamics
}

\author{
Sharma S.R.K.C. Yamijala, ${ }^{1, \#}$ Zulfikhar A. Ali, ${ }^{2, \#}$ and Bryan M. Wong ${ }^{1,2,3,{ }^{*}}$ \\ ${ }^{1}$ Department of Chemical \& Environmental Engineering, ${ }^{2}$ Department of Physics \& Astronomy \\ and ${ }^{3}$ Materials Science \& Engineering Program, University of California-Riverside, Riverside, \\ California 92521, United States \\ \# These authors have contributed equally \\ *E-mail: bryan.wong@ucr.edu, Webpage: http://www.bmwong-group.com
}

\section{Abstract}

$\mathrm{Ab}$ initio molecular dynamics (AIMD) is an indispensable tool for understanding the mechanistic details of externally-energy mediated chemical reactions. In this work, we show that the predicted thermodynamic and catalytic properties of certain reactions using AIMD simulations critically depend on the quality of the employed basis set. To this end, we have examined the reactants and products of the water-gas shift reaction (viz., $\mathrm{CO}, \mathrm{CO}_{2}, \mathrm{H}_{2}$, and $\mathrm{H}_{2} \mathrm{O}$ ) and studied their interaction with the $\mathrm{ZnO}(1010)$ surface using density functional theory (DFT) and Born Oppenheimer Molecular Dynamics (BOMD) simulations. By merely increasing the quality of the basis, from double zeta (commonly used in most calculations of these systems) to triple zeta, we surprisingly find that the reaction outcome of an $\mathrm{H}_{2} \mathrm{O}$ molecule colliding with a ZnO surface pre-covered with carbon monoxide gives qualitatively different results. These surprising results are shown to be robust with similar trends that are also obtained with other software packages. Furthermore, we show that the calculated adsorption energies can vary by as much as 380 meV (which is an order of magnitude larger than room temperature) by simply changing the basis set. Using electron density difference maps, we present mechanistic insight into the origin of these changes. Finally, we propose a simple diagnostic test that uses a single-point binding energy calculation to estimate the impact of basis-set quality, which can be used before carrying out a computationally-expensive BOMD simulation.

\section{Introduction}

The channeling of energy via "hot" electrons and thermally-excited atoms/molecules towards a specific reaction channel has recently emerged as a new area in the catalysis community for controlling and understanding the mechanism and dynamics of catalytic reactions. $\stackrel{1-11}{=}$ Predicting the reaction mechanisms of these externally-energy mediated reactions using traditional quantum chemical approaches, where the energies of the reactants, products, and transition states are used to predict the reaction mechanisms, is nontrivial. $\stackrel{12-15}{ }$ This complexity arises due to various reasons, such as difficulties pertaining to the determination of 
transition states, requirements in considering numerous configurations for the adsorbate, the formation of complex transition states involving more than two molecules, etc. ${ }^{12.13}$ Apart from this complexity, typical computational results are obtained only at zero Kelvin, and extrapolations of these results to realistic reaction temperatures are questionable. An alternative, automatic, and unbiased approach to predict the mechanism of a reaction is to use ab initio molecular dynamics (AIMD). By its nature, an AIMD simulation automatically includes the dynamic and steric effects of a reaction with a simultaneous prediction of possible reaction mechanisms. $\frac{12,13}{\underline{1}}$

Among the various AIMD methods, Born Oppenheimer Molecular Dynamics (BOMD) has been quite successful in predicting the mechanisms and possible outcomes of reactions involving atomic or molecular collisions. $\frac{12,13,16-25}{1}$ For example, recently Schatz and co-workers have used BOMD simulations to understand the detailed mechanisms of industrially relevant reactions such as the reverse water-gas shift reaction and Fischer-Tropsch synthesis on nickel surfaces, ${ }^{18,20}$ where the pre-coated $\mathrm{Ni}$ surface was bombarded with energetic hydrogen atoms and methylene, respectively. Wang et. al., ${ }^{17}$ have used BOMD to probe the water dissociation equilibrium on $\mathrm{TiO}_{2}$ by colliding energetic water molecules towards the $\mathrm{TiO}_{2}$ surface. BOMD has also been employed in predicting the reaction outcomes of atmospherically relevant molecular collisions such as the formation of carbonic acid obtained by the collision between a $\mathrm{CO}_{2}$ molecule and $\mathrm{H}_{2} \mathrm{O}$ clusters, $\underline{25}$ and the formation of sulphuric acid $\frac{12.13}{2}$ via the oxidation of $\mathrm{SO}_{2}$ to $\mathrm{SO}_{3}$, resulting from a collision between $\mathrm{SO}_{2}$ and $\mathrm{O}_{3}{ }^{-}\left(\mathrm{H}_{2} \mathrm{O}\right)_{n}$ clusters.

Considering the previously mentioned successes of the BOMD method with collision reactions, it is both important and necessary to study the specific computational parameters that can alter the mechanism and dynamics predicted by this method. Both the mechanism and dynamics of a chemical reaction are dictated by the forces acting on the nuclei. In BOMD, these forces are obtained in an ab initio manner from the ground state potential, which in turn is obtained with electronic structure methods such as Hartree-Fock, DFT, DFTB, etc. Furthermore, as density functional theory (DFT) is the most widely-used approach for calculating the electronic structure during a BOMD simulation, $\frac{26-28}{28}$ and since DFT results are sensitive to various parameters such as basis-sets and exchange-correlation functionals, the resulting BOMD dynamics is also expected to be affected by these factors. However, the effect of these parameters on the predicted results of a BOMD simulation has not been well studied, and in the present work we focus our attention on one of these parameters, namely, the importance of the basis set.

It is worth noting that while there are numerous studies on the effect of basis sets on ground state (i.e. stationary) structures, $\frac{29-32}{}$ there are only a handful of studies on basis set effects in BOMD simulations. $\frac{33-39}{2}$ Furthermore, most of these studies were devoted to understanding basis set effects in clusters. Thus, to the best of our knowledge, an in-depth study of basis set effects in large-scale Born-Oppenheimer molecular dynamics (BOMD) calculations (with periodic boundary conditions) is less common. Such investigations have not been widely carried out since the conventional assumption in these studies is that double-zeta (DZ) quality basis sets are assumed to work well, $\stackrel{12,17,23-25}{ }$ and comparing the BOMD calculations with larger basis-sets is quite computationally demanding. Although the above assumption may 
seem reasonable, we demonstrate in this work that calculations using the widely-used double zeta basis can yield completely different results than those obtained with a larger basis (for example, a triple-zeta (TZ) quality basis). As shown in our calculations, the reaction mechanisms obtained from BOMD simulations using DZ basis sets may need to be reevaluated. For example, the dissociation equilibrium of water predicted by Wang et. al., ${ }^{17}$ depends on whether the water molecule colliding with the $\mathrm{TiO}_{2}$ dissociates on the surface or whether it scatters from the surface. However, as shown in this work, the fate of a colliding molecule highly depends on the employed basis set, which can qualitatively change the entire prediction.

In the present work, we have studied the collision of an $\mathrm{H}_{2} \mathrm{O}$ molecule with a $\mathrm{ZnO}$ surface pre-covered with carbon monoxide with both double- and triple-zeta quality basis sets using BOMD simulations. To gain further insight, we have also studied the interaction of $\mathrm{CO}$, $\mathrm{CO}_{2}, \mathrm{H}_{2}$, and $\mathrm{H}_{2} \mathrm{O}$ with the $\mathrm{ZnO}$ surface with both of these basis sets. Based on the results from both BOMD and DFT, we propose that single-point binding energy calculations can be used as a simple diagnostic tool to estimate the impact of basis-set quality before carrying out a computationally-expensive BOMD simulation. Finally, we note that our simulations are relevant to the industrially-relevant water-gas shift reaction, where $\mathrm{ZnO}$ is one of the co-catalysts, and all of the above-mentioned molecules are either products or reactants of this reaction. $\frac{40-44}{4}$

\section{Computational Details}

All calculations were performed with Kohn-Sham density functional theory using the $\mathrm{PBE}^{45}$ exchange-correlation functional as implemented in the CP $2 \mathrm{~K}, \underline{26}$ FHI-aims,,$\underline{27}$ and VASP $\underline{28}$ software packages. In $\mathrm{CP} 2 \mathrm{~K}$, we have used both the molecularly optimized double-zeta quality (DZVP) and triple-zeta quality (TZV2P) basis-sets, $\frac{46}{,}$ which are compatible with the employed Goedecker-Teter-Hutter (GTH) pseudopotentials. ${ }^{47.48}$ For the auxiliary plane-wave (PW) basis, used in the Gaussian-and-Plane-Waves (GPW) method of CP2K, we have used 1000 Ry for the PW energy cutoff and 60 Ry for the reference grid cutoff. In FHI-aims, we have used both the Tier-1 and Tier-2 numerical orbital basis-sets. In VASP, we have used the default plane-wave basis with a $500 \mathrm{eV}$ energy cutoff. We would like to point out that the aim of this study is not to judge the accuracy of the exchange-correlation functional; rather, we highlight the role of the basis set in predicting the catalytic dynamics (mechanistic outcomes) of a reaction. As such, we have only considered one of the most widely used exchange-correlation functionals, namely, PBE (although we anticipate that the dramatic basis set effects shown in this work will also apply to other functionals).

For all of the electronic structure calculations, a $4 \times 4$-slab with a thickness of 4 layers was used. A vacuum of at least $15 \AA$ was used to avoid any spurious interactions between the periodic images. Since the choice of a k-mesh did not affect our findings (see Supporting Information), we only present calculations performed at the $\Gamma$-point of the Brillouin zone. Furthermore, since recent experiments have shown that $\mathrm{CO}$ molecules can easily hop along the a-direction of the $\mathrm{ZnO}$ slab (due to the relatively smaller lattice spacing along that direction), ${ }^{49}$ in 
all of our BOMD simulations, we have expanded our slab along the a-direction, to capture this hopping mechanism. Thus, in all of our NVE and NVT calculations performed with CP2K, we have used a $6 \times 3$ slab with a 4-layer thickness (instead of a $4 \times 4$-slab). We have integrated the equations of motion with a 0.5 fs time-step. The initial velocities and coordinates for the NVE runs were obtained by running an NVT simulation at $300 \mathrm{~K}$. For the NVT simulations, we have used the Nosé-Hoover thermostat of chain length three. In all the NVT and NVE runs, Grimme's D3-dispersion correction was employed. $\underline{50}$ Various other computational details are given in the Supporting Information.

\section{Results and Discussion}

Figure 1a shows the initial configuration used to study the interaction of an $\mathrm{H}_{2} \mathrm{O}$ molecule colliding with a $\mathrm{ZnO}$ slab pre-adsorbed with $\mathrm{CO}$ molecules at $0.22 \mathrm{ML}$ coverage. For simplicity, we have only considered the normal incidence of $\mathrm{H}_{2} \mathrm{O}\left(90^{\circ}\right.$ to the slab). We have studied the impact of $\mathrm{H}_{2} \mathrm{O}$ with two incident energies, namely $0.6 \mathrm{eV}$ and $6 \mathrm{eV}$. Here, the lower incident energy of $0.6 \mathrm{eV}$ was chosen since it is slightly above the binding energy of the CO molecule on a ZnO surface $(0.26-0.53 \mathrm{eV})$, obtained at the PBE level of theory. ${ }^{49.51}$ In other words, it is the minimum amount of energy required for the $\mathrm{CO}$ molecule to hop or diffuse on the ZnO surface. To represent the high-energy collision, we used a $6 \mathrm{eV}$ incidence, which is an order of magnitude larger than the binding energy of the adsorbate. 

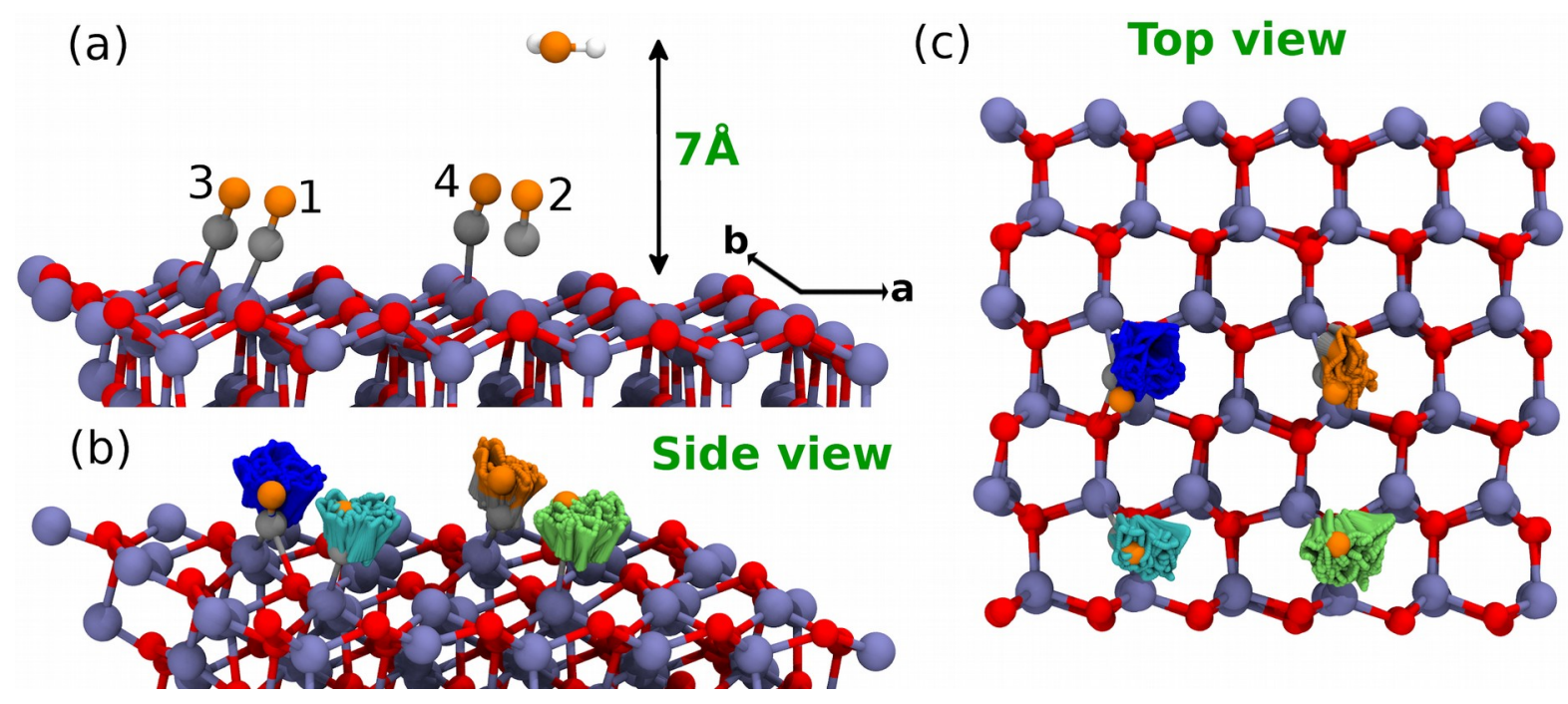

Figure 1: (a) Initial configuration used to study the interaction of an $\mathrm{H}_{2} \mathrm{O}$ molecule colliding with a $\mathrm{ZnO}$ slab preadsorbed with four $\mathrm{CO}$ molecules. The four $\mathrm{CO}$ molecules are numbered to distinguish the $4^{\text {th }} \mathrm{CO}$ molecule (closest one to the impact region) from the others. The blue, red, grey, and white colors are used to represent the $\mathrm{Zn}, \mathrm{O}, \mathrm{C}$, and $\mathrm{H}$ atoms, respectively. Oxygen atoms of the $\mathrm{CO}$ and $\mathrm{H}_{2} \mathrm{O}$ are colored in orange to distinguish them from the oxygen atoms of the ZnO lattice. Panels (b) and (c) show the side and top views of how the CO molecules in configuration (a) have changed during a 4.4 ps NVT run performed with the TZV2P basis sets at $300 \mathrm{~K}$. In both (b) and (c), the cyan, lime, and blue trajectories show the change in the positions of $1^{\text {st }}, 2^{\text {nd }}$, and $3^{\text {rd }}$ CO molecules, respectively, for the entire trajectory.

Before proceeding to the collision reaction results, we would first like to show how the adsorbed CO molecules evolve during an NVT simulation (performed at $300 \mathrm{~K}$ and with the TZV2P basis) in the absence of any collision. In figure 1 b, we have shown the positions of each of the $\mathrm{CO}$ molecules for every 10 fs during the entire trajectory. Clearly, throughout the $4.4 \mathrm{ps}$ simulation time, all of the CO molecules remained in their binding sites and fluctuated around their mean positions. Apart from these fluctuations, we did not find any significant changes (such as a hop, diffusion, or desorption of any $\mathrm{CO}$ ) during the simulation. Similar results were also obtained with the DZVP basis (see figure S11). Next, we present the effect of basis set quality on the collision reactions.

\section{Low-energy $(0.6 \mathrm{eV})$ collision}

We now present our NVE simulation results of a water molecule colliding with the ZnO surface with a $0.6 \mathrm{eV}$ translational energy. In figure $2 \mathrm{a}$ and $\underline{2} \mathrm{~b}$, we show the final configuration obtained after a 3 ps simulation performed with the double zeta (DZVP) and triple zeta (TZV2P) basis, respectively. The relative change in the positions of the $4^{\text {th }} \mathrm{CO}$ and $\mathrm{H}_{2} \mathrm{O}$, along the adirection of the lattice, for the entire trajectory is shown in figure $2 \mathrm{c}$. The changes depicted here are relative to the initial frame (shown in figure 1a). Here, we would like to note that there is no head-on collision of the $\mathrm{H}_{2} \mathrm{O}$ with the $4^{\text {th }} \mathrm{CO}$. However, as it is the closest one to the impact area, it has the maximum effect. Also, hereafter, we denote the TZV2P basis as TZ, and the DZVP basis is denoted as DZ. 

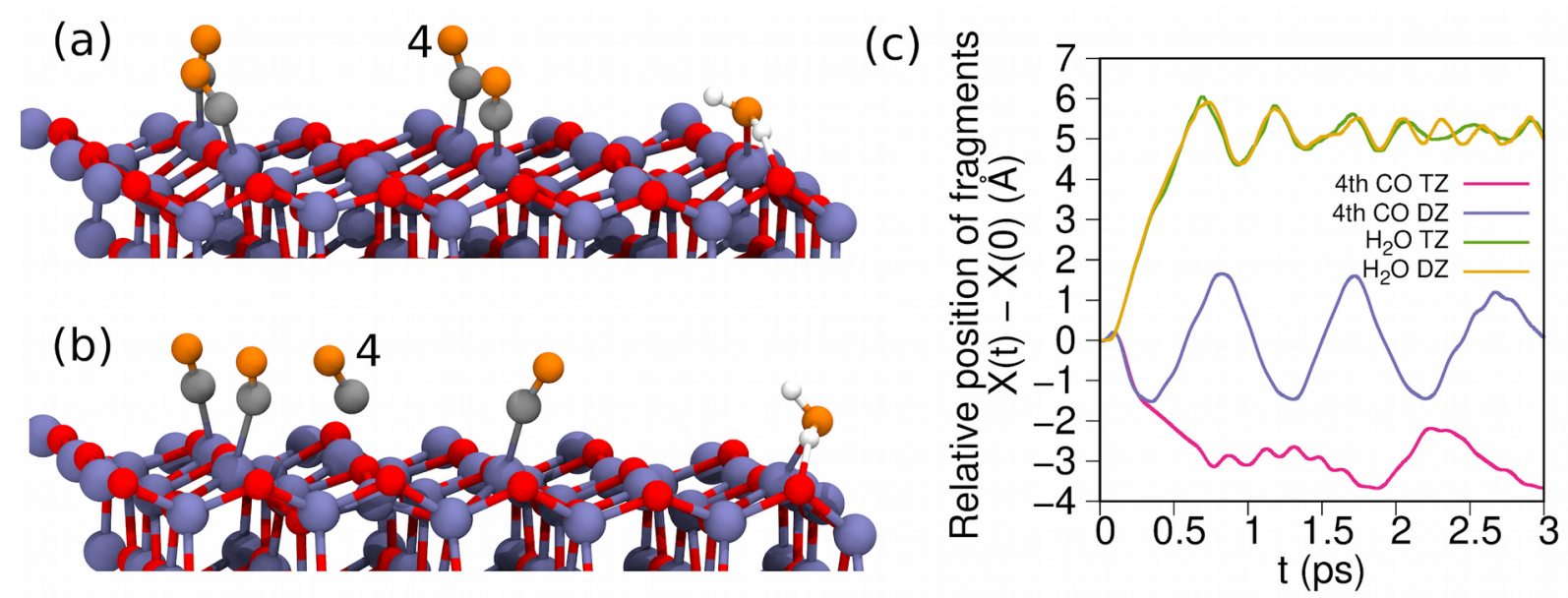

Figure 2: Panels (a) and (b) show how the configuration in figure 1a has changed for a $0.6 \mathrm{eV}$ incident energy for a $\mathrm{H}_{2} \mathrm{O}$ molecule after a 3 ps NVE simulation performed with the DZVP and TZV2P basis sets, respectively. (c) Relative change in the positions of the $4^{\text {th }} \mathrm{CO}$ and $\mathrm{H}_{2} \mathrm{O}$ molecules along the a-direction of the lattice.

As shown in figure $2 \mathrm{a}$ and figure $\mathrm{S} 12 \mathrm{a}$, with the $\mathrm{DZ}$ basis, none of the CO molecules have left their binding sites even after 3 ps of simulation time. Furthermore, during the entire simulation time, their relative positions (only shown here for the $4^{\text {th }} \mathrm{CO}$ ) were merely oscillating around zero (figure 2c, blue curve). This oscillatory behavior around zero corresponds to the thermal fluctuations around their initial positions. Thus, the low-energy collision did not result in any significant changes in the behavior of the $\mathrm{CO}$ molecules on the $\mathrm{ZnO}$ surface. On the other hand, with the TZ basis, we observed a hop of the $4^{\text {th }} \mathrm{CO}$ from one $\mathrm{Zn}$ site to the other one (see figure $2 \mathrm{~b}$ and figure S12b as compared with figure 1a). As shown in figure $2 \mathrm{c}$, this hop actually occurred within the first picosecond of the simulation time and is manifested by a decrement in the relative position of the $4^{\text {th }} \mathrm{CO}$ by $\sim 3$ units in the negative direction. Here, $\sim 3 \AA$ is the distance between two $\mathrm{Zn}$ sites along the a-direction (3.28 $\AA$, for the ground state structure), and the negative change corresponds to the hop along the negative a-direction of the lattice. After this hop, the $4^{\text {th }} \mathrm{CO}$ continued to remain at the new $\mathrm{Zn}$ site while exhibiting thermal fluctuations (figure 2c and figure S12b). Finally, the positive change in the position of the $\mathrm{H}_{2} \mathrm{O}$ with both basis sets corresponds to its hop along the positive a-direction of the lattice. This change in the position of the $\mathrm{H}_{2} \mathrm{O}$ and its adsorption onto the $\mathrm{ZnO}$ surface (after the collision) can be seen in both figure $2 \mathrm{a}$ and $\underline{2} \mathrm{~b}$. 

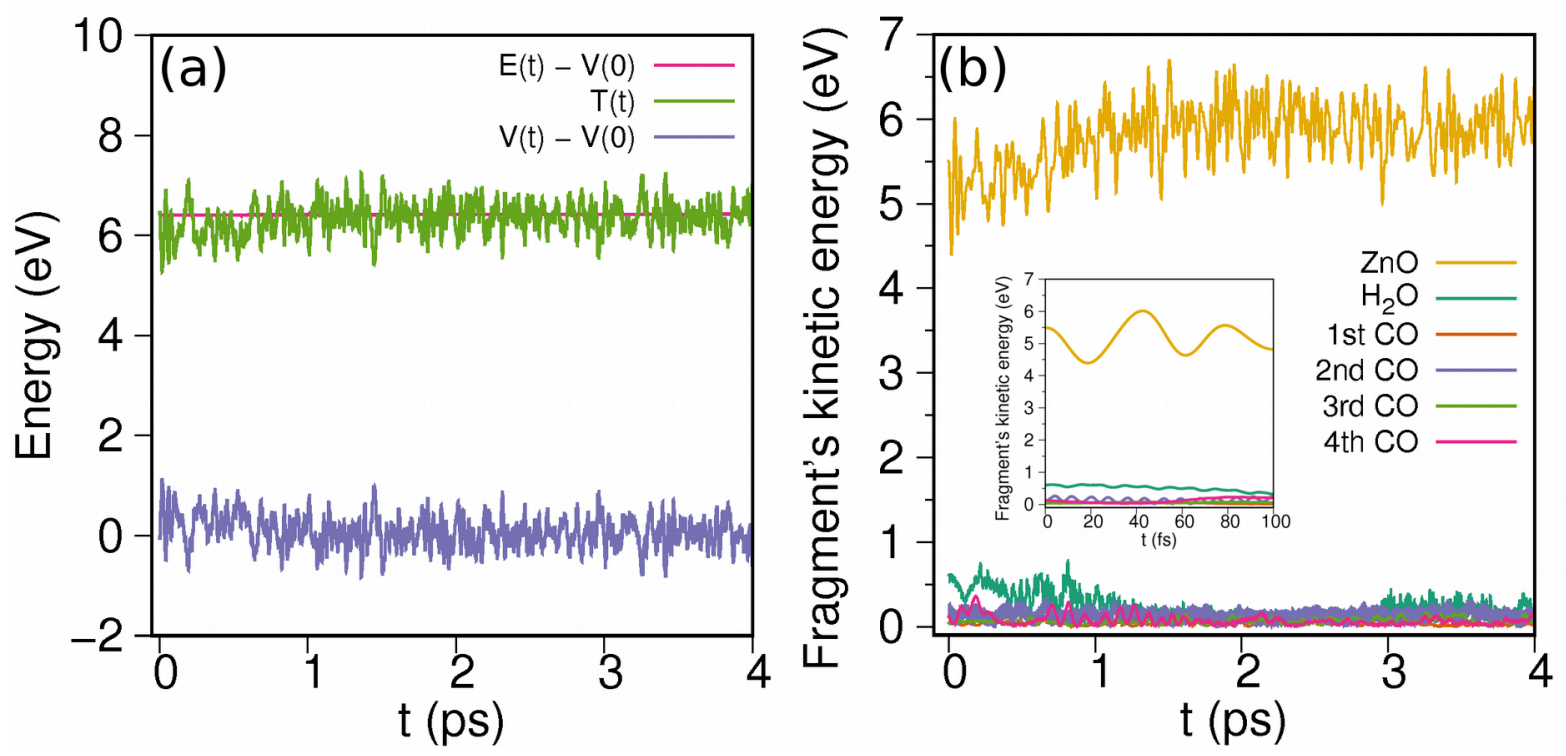

Figure 3: Panel (a) shows the changes in total $(E)$, kinetic $(T)$, and potential $(V)$ energies of the entire system for the $0.6 \mathrm{eV}$ collision simulated with the TZ basis. Panel (b) shows the changes in the kinetic energy of each of the fragments during the same NVE run. The inset in (b) shows the changes in kinetic energies of the fragments during the first $100 \mathrm{fs}$ of the simulation.

Next, we discuss the changes in the energetics of this $0.6 \mathrm{eV}$ collision reaction. In figure $\underline{3} a$, we have given the total $(E)$, kinetic ( $T$ or KE), and potential ( $V$ or PE) energies of the entire system during the NVE run with the TZ basis. Clearly, the total energy of the entire system is conserved, demonstrating the NVE nature of this simulation. Also, due to the low-impact energy, there are only minor changes to the total KE or PE of the system. As shown in figure $3 b$, the incident $\mathrm{H}_{2} \mathrm{O}$ molecule transferred some $(\sim 0.3 \mathrm{eV})$ of its $\mathrm{KE}$ towards the $4^{\text {th }} \mathrm{CO}$ molecule during the initial stages of the run ( $<1 \mathrm{ps})$, resulting in the hopping of the $4^{\text {th }} \mathrm{CO}$ from one $\mathrm{Zn}$ site to the other (as discussed earlier). At a later time, the KE of both the $\mathrm{H}_{2} \mathrm{O}$ and the $4^{\text {th }} \mathrm{CO}$ has been completely transferred to the $\mathrm{ZnO}$ slab. This energy transfer between fragments can be clearly noticed in figure $3 \mathrm{~b}$ as the rise in the KE of the $\mathrm{ZnO}$ and the decay of the $\mathrm{H}_{2} \mathrm{O}$ and $4^{\text {th }} \mathrm{CO}$ molecule's KE after $\sim 2$ ps simulation time. During the rest of the simulation time, all of the molecules (four COs and one $\mathrm{H}_{2} \mathrm{O}$ ) remained vibrating around their mean positions. Thus, for $0.6 \mathrm{eV}$ of collision energy, during the entire run, we only observed hopping of the $4^{\text {th }} \mathrm{CO}$ and the adsorption of $\mathrm{H}_{2} \mathrm{O}$ to the $\mathrm{ZnO}$ surface. The energetics of the simulation with the DZ basis are given in figure $\mathrm{S} 13$.

\section{High-energy (6 eV) collision}

Next, we present the results of the high-energy collision ( $6 \mathrm{eV})$. In figure $4 \mathrm{a}$ and $4 \mathrm{~b}$, we show the final configuration obtained after a 5 ps NVE simulation performed with the DZ and TZ basis, respectively. First, unlike the low-energy collision, we observed the dissociation of the colliding $\mathrm{H}_{2} \mathrm{O}$ molecule into $\mathrm{OH}$ and $\mathrm{H}$ species, with both basis sets. Also, we find that the dissociated $\mathrm{OH}$ and $\mathrm{H}$ species were within the bonding region of the $\mathrm{Zn}$ and $\mathrm{O}$ sites of the $\mathrm{ZnO}$ lattice, 
respectively. Unlike the case of $\mathrm{H}_{2} \mathrm{O}$, we obtained significantly different results for the $4^{\text {th }} \mathrm{CO}$ with the DZ and TZ basis. After a 5 ps simulation with the TZ basis, we find the complete desorption of the $4^{\text {th }} \mathrm{CO}$ (figure $4 \mathrm{~b}$ ); however, with the DZ basis, we only find the diffusion of the $4^{\text {th }} \mathrm{CO}$ (figure $\left.4 \mathrm{a}\right)$.

(a)

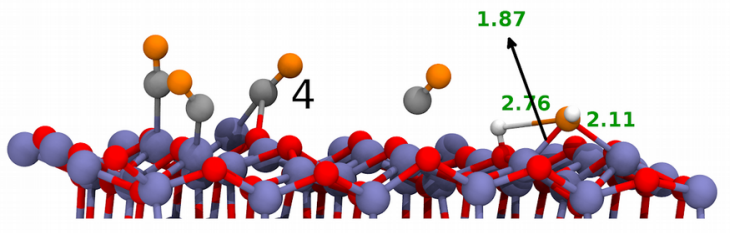

(b)

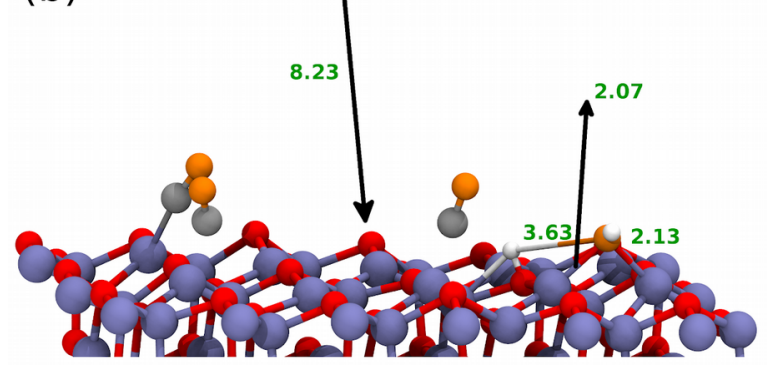

(c)

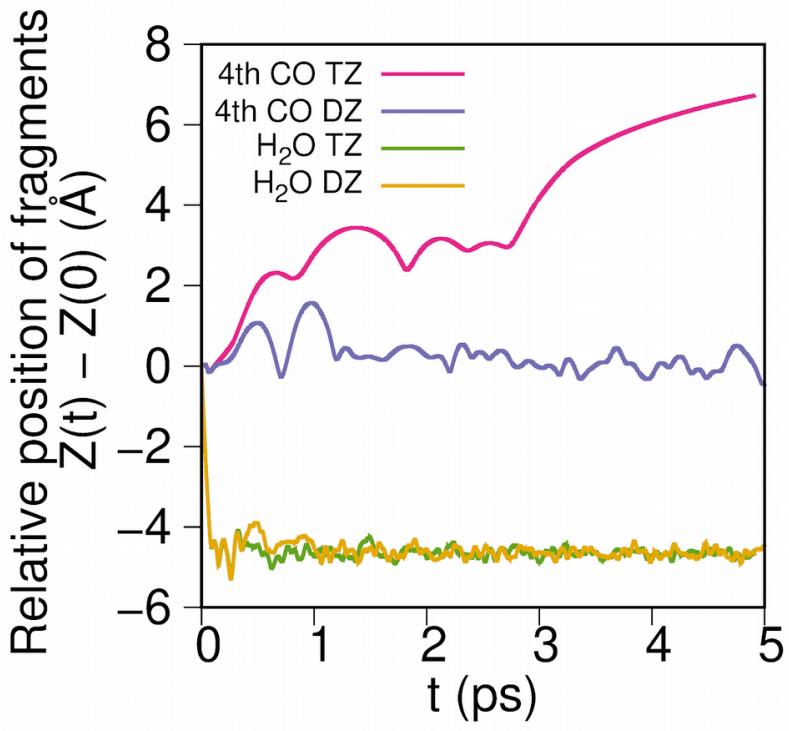

Figure 4: Panels (a) and (b) show how the configuration in figure 1a has changed for the $6 \mathrm{eV} \mathrm{H}_{2} \mathrm{O}$ incident energy, after a 5 ps NVE simulation performed with the DZVP and TZV2P basis sets, respectively. (c) Relative change in the positions of the $4^{\text {th }} \mathrm{CO}$ and $\mathrm{H}_{2} \mathrm{O}$ molecules along the c-direction of the lattice.

In figure $4 \mathrm{c}$, we present the relative change in the positions of the $4^{\text {th }} \mathrm{CO}$ and $\mathrm{H}_{2} \mathrm{O}$, along the c-direction of the lattice, for the entire trajectory. Once again, the depicted changes are relative to the initial frame (shown in figure 1a). Here, for the $\mathrm{H}_{2} \mathrm{O}$, a relative position of zero Angstroms corresponds to its initial state of $\sim 6.5 \AA$ above the surface and a relative position of $-5 \AA$ corresponds to its contact with the $\mathrm{ZnO}$ surface. Thus, as shown in figure $4 \mathrm{c}$, the $\mathrm{H}_{2} \mathrm{O}$ molecule made contact with the $\mathrm{ZnO}$ surface within $\sim 80 \mathrm{fs}$, for both basis sets and remained on the surface until the end of the simulation. On the other hand, the $4^{\text {th }} \mathrm{CO}$ showed qualitatively different results with both basis sets. In this figure, for the $4^{\text {th }} \mathrm{CO}$, a relative position of zero Angstroms corresponds to its initial adsorption configuration on the $\mathrm{ZnO}$ surface, and a relative position of greater than $6 \AA$ corresponds to its complete desorption from the ZnO surface. As such, with the TZ basis, we observed an initial dissociation ( $>1 \AA$ ) of the $4^{\text {th }} \mathrm{CO}$ from its $\mathrm{Zn}$ binding site in less than a picosecond (figure 4c) followed by its diffusion on the $\mathrm{ZnO}$ surface $(\sim 2-3 \AA)$ until $\sim 3 \mathrm{ps}$, and finally a complete desorption from the $\mathrm{ZnO}$ surface at $\sim 4 \mathrm{ps}$ of the run. On the other hand, with the DZ basis, the $\mathrm{CO}$ always remained below $2 \AA$ from the $\mathrm{ZnO}$ surface, suggesting a diffusion-like behavior. As noted earlier, for the TZ basis calculation, the CO molecule was also diffusing during the first 3 ps simulation time, albeit at a larger distance $(>2$ $\AA$ ) from the $Z n O$ surface compared to the $\mathrm{CO}$ when the $\mathrm{DZ}$ basis was used. A visual representation of these changes for the entire trajectory is given in figure S14. 

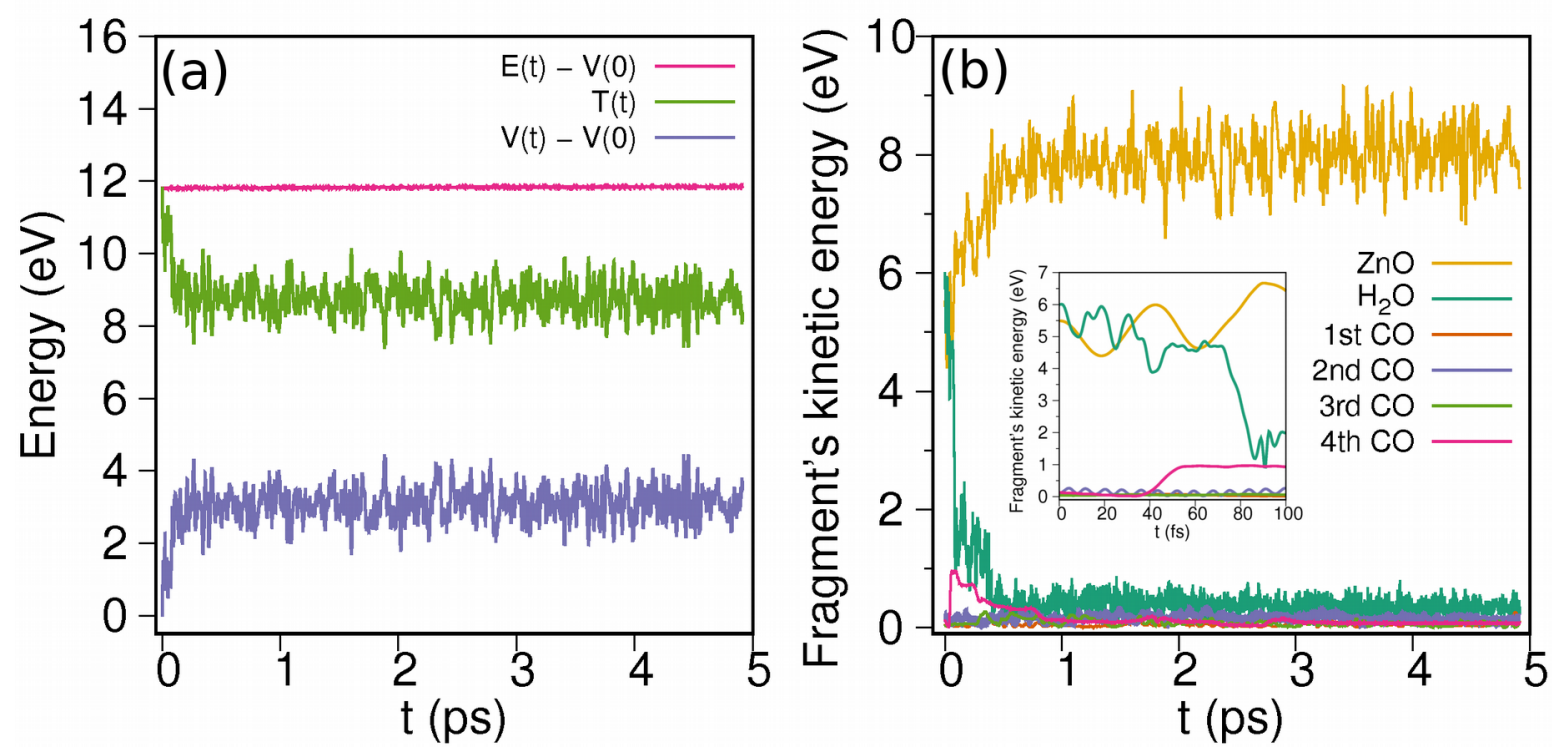

Figure 5: Panel (a) shows the changes in total $(E)$, kinetic $(T)$, and potential $(V)$ energies of the entire system for the $6 \mathrm{eV}$ collision simulated with the TZ basis. Panel (b) shows the changes in the kinetic energy of each of the fragments during the same NVE run. The inset in (b) shows the changes in kinetic energies of the fragments during the first 100 fs of the simulation.

Next, in figures $5 \mathrm{a}$ and $\underline{5} \mathrm{~b}$, we present the energy profiles of the $6 \mathrm{eV}$ collision, with the TZ basis. Once again, the total energy of the entire system is conserved. However, unlike the low-energy collision, the changes in the KE or PE of the system are quite apparent for the $6 \mathrm{eV}$ collision. Here, we find an ultrafast (<200 fs) decay (rise) in the total kinetic energy (potential energy) of the system, as shown in figure $5 \mathrm{a}$. To understand this ultrafast decay in the total KE, we have plotted the changes in the $\mathrm{KE}$ of the individual fragments in figure $5 \mathrm{~b}$. Clearly, the ultrafast decay of the total $\mathrm{KE}$ can be directly correlated with the ultrafast decay in the $\mathrm{H}_{2} \mathrm{O}$ molecule's KE (inset of figure $5 \mathrm{~b}$ ). However, although the $\mathrm{H}_{2} \mathrm{O}$ molecule has lost most of its KE $(\sim 5 \mathrm{eV})$ in less than $100 \mathrm{fs}$, only a part of it $(\sim 3 \mathrm{eV})$ has been transferred to the other fragments ( $\sim 1 \mathrm{eV}$ to the 4 th $\mathrm{CO}$ and $\sim 2 \mathrm{eV}$ to the $\mathrm{ZnO}$ lattice) in the form of KE (see figure $5 \mathrm{~b}$ ). The rest is transformed into the PE of the system leading to its increase. The reason for such a rise in the $\mathrm{PE}$ of the system is due to the formation and dissociation of various bonds during the first $200 \mathrm{fs}$ of the run. At $\sim 80 \mathrm{fs}$, the $\mathrm{H}_{2} \mathrm{O}$ molecule made contact with the $\mathrm{ZnO}$ surface (see figure $4 \mathrm{c}$ and figure S15), and subsequently the dissociation process of water was initiated. Following the water dissociation (into $\mathrm{OH}$ and $\mathrm{H}$ ) we observed the formation of $\mathrm{Zn}-\mathrm{OH}$ and $\mathrm{O}-\mathrm{H}$ bonds with the $\mathrm{Zn}$ and $\mathrm{O}$ atoms of the slab, respectively (see figure S15). Also, during this time, due to the increased KE of the $4^{\text {th }} \mathrm{CO}$, the $\mathrm{Zn}-\mathrm{CO}$ bond subsequently dissociated (see figure $4 \mathrm{c}$ and figure $\mathrm{S} 15)$. Thus, the ultrafast decay in the KE profile is due to a combination of many factors as explained above. We have given the energetics of the same $6 \mathrm{eV}$ collision with the DZ basis in figure S16. 


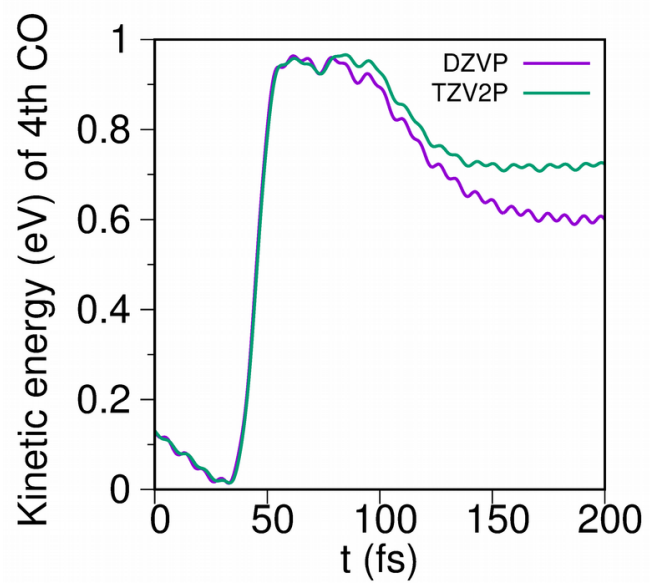

Figure 6: Similar results in the rise of the KE of the $4^{\text {th }} \mathrm{CO}$ using both the DZ and TZ basis during the first $100 \mathrm{fs}$ of the simulation.

We would like to emphasize here that the simulations with both the TZ and DZ basis behaved almost similarly during the $0-200$ fs simulation time. This similarity is apparent both in their energetics and dynamics (compare figure 5 and figure S16; also see figure S15). The differences between the TZ and DZ basis start after $\sim 200 \mathrm{fs}$, and as noted earlier, the differences are quite apparent in the dynamics of the $4^{\text {th }} \mathrm{CO}$. However, even for the $4^{\text {th }} \mathrm{CO}$, the energetics and dynamics are quite similar with the DZ and TZ basis during the first few hundred femtoseconds as shown in figure 6 and figure $4 \mathrm{c}$. Clearly, both the KE gain by the $4^{\text {th }} \mathrm{CO}$ (figure 6) and its relative position (figure 4c) are almost the same, until $\sim 200 \mathrm{fs}$ for both the DZ and TZ runs (also see figure S15). Thus the differences observed in the dynamics of the $4^{\text {th }} \mathrm{CO}$ are not due to any changes in its $\mathrm{KE}$ gain (from $\mathrm{H}_{2} \mathrm{O}$ ), but only due to the difference in the interactions among various fragments of the system introduced by the employed basis set. In the next section, we study how the interaction between the adsorbate molecule and the ZnO surface vary with a change in the basis set.

Until now, we have found that irrespective of the $\mathrm{H}_{2} \mathrm{O}$ 's incident energy, we have always obtained different results using the $\mathrm{DZ}$ and $\mathrm{TZ}$ basis-sets for the same initial configuration and for the same initial rise (loss) in the KE energy by the $4^{\text {th }} \mathrm{CO}\left(\mathrm{H}_{2} \mathrm{O}\right)$. While the differences are just noticeable in the case of the $0.6 \mathrm{eV}$ collision, they are drastically different for the case of the high-energy collision. For the $6 \mathrm{eV}$ collision, with the DZ basis, we have only observed the diffusion of the $4^{\text {th }} \mathrm{CO}$ on the $\mathrm{ZnO}$ surface, whereas we observed a qualitatively different mechanism for the TZ basis - a complete desorption (apart from the initial diffusion). Furthermore, for the $6 \mathrm{eV}$ collision, the differences in the reaction outcomes between the DZ and TZ basis were found to be persistent with a change in the impact position (verified by impacting the $\mathrm{H}_{2} \mathrm{O}$ molecule in the vicinity of the $2^{\text {nd }} \mathrm{CO}$ molecule instead of the $4^{\text {th }} \mathrm{CO}$ molecule) and with the inclusion/neglect of dispersion corrections. These additional results are presented in the Supporting Information. This significant difference in the collision-reaction outcomes obtained by merely changing the basis is one of the central findings of our work. 


\section{Effect of basis set on the adsorption energies}

To further understand the reason for this difference, we have performed a few adsorption energy calculations with different adsorbates on the ZnO surface. In all of these calculations, we have calculated the adsorption energy $\left(E_{a d s}\right)$ of a molecule on a $\mathrm{ZnO}$ slab as

$$
E_{\text {ads }}=E_{(\text {slab+mol })}-E_{\text {slab }}-E_{m o l}
$$

where, $E_{(\text {slab+mol) }}$ is the energy of the entire system (slab + adsorbate molecule), $E_{\text {slab }}$ is the energy of the $\mathrm{ZnO}$ slab, and $\mathrm{E}_{\mathrm{mol}}$ is the energy of the adsorbate molecule.

Table 1: Adsorption energies of the molecules calculated using CP2K with different basis sets and differences in the adsorption energies between basis sets.

\begin{tabular}{|c|c|c|c|}
\hline System & $\begin{array}{c}\text { Adsorption } \\
\text { energy with the } \\
\text { DZVP basis, } \\
\mathrm{E}_{\text {DZVP }}(\mathrm{eV})\end{array}$ & $\begin{array}{c}\text { Adsorption } \\
\text { energy with the } \\
\text { TZV2P basis, } \\
\mathrm{E}_{\text {TZV2P }}(\mathrm{eV})\end{array}$ & $\begin{array}{c}\text { Difference in adsorption } \\
\text { energy, } \\
\mathrm{E}_{\text {TZV2P }}-\mathrm{E}_{\text {DZVP }}(\mathrm{eV})\end{array}$ \\
\hline ZnO Slab + CO & -0.884 & -0.504 & 0.380 \\
\hline ZnO Slab + CO2 & -0.177 & -0.175 & 0.002 \\
\hline ZnO Slab + H2 & -0.088 & -0.083 & 0.005 \\
\hline ZnO Slab + H2O & -0.990 & -0.961 & 0.029 \\
\hline
\end{tabular}

In table 1, we have given the adsorption energies of all four molecules calculated using CP2K with both the $\mathrm{DZ}$ and $\mathrm{TZ}$ basis. We have also given the difference in the adsorption energy ( $E_{\text {diff }}{ }^{T D}$ ) between the $T Z$ and $D Z$ basis. Except for $C O$, the adsorption energy of all other molecules is not sensitive to basis set effects (differences are on the order of room temperature). However, surprisingly, for $\mathrm{CO}$ the change in the adsorption energy with a change in the basis is more than an order of magnitude $(\sim 0.4 \mathrm{eV})$ larger than room-temperature $(\sim 0.026$ $\mathrm{eV}$ ). This huge difference in the $\mathrm{CO}$ adsorption energies with a change in the basis is the primary reason for the completely different results observed in the collision calculations (as shown in the earlier sections) despite their similarities during the initial stages of the run. From the adsorption energies, it is clear that $\mathrm{CO}$ is weakly bound to the $\mathrm{ZnO}$ substrate (by $\sim 0.4 \mathrm{eV}$ ) when using the TZ basis. We note that this weakness in the interaction between $\mathrm{ZnO}$ and $\mathrm{CO}$ with the TZ basis manifests itself in both the easy dissociation and hopping of the $4^{\text {th }} \mathrm{CO}$ for the $0.6 \mathrm{eV}$ collision and the complete desorption of the $4^{\text {th }} \mathrm{CO}$ for the $6 \mathrm{eV}$ collision. Neither the hop nor the desorption of the $\mathrm{CO}$ was observed while using the DZ basis because of the stronger interaction between $\mathrm{CO}$ and $\mathrm{ZnO}$ with this basis set. This difference in the interaction between $\mathrm{CO}$ and $\mathrm{ZnO}$ with a change in the basis can also be noticed in the shorter (longer) distance between $\mathrm{CO}$ and $\mathrm{ZnO}$ during the diffusion of $\mathrm{CO}$ on the surface with the $\mathrm{DZ}$ (TZ) basis. 


\section{Electron density difference maps}

To gain further insight into the influence of the basis set on the interaction between the slab and the adsorbate molecule, we have plotted the electron density differences as shown in figure 7. First, for each basis set, we have computed the electron density difference ( $\rho$ [diff]) as

$$
\rho[\text { diff }]=\rho[Z n O+m o l]-\rho[Z n O]-\rho[m o l]
$$

where, $\rho[\mathrm{ZnO}+\mathrm{mol}], \rho[\mathrm{ZnO}]$, and $\rho[\mathrm{mol}]$ are the electron densities of the entire system (slab + adsorbate molecule), ZnO slab, and adsorbate molecule, respectively. Here, all the $\rho$ [diff] plots are generated by fixing the fragment geometries to the optimized geometry of the entire system (optimized at the TZV2P/PBE level of theory). These electron density differences ( $\rho$ [diff]) using both the DZ and TZ basis are shown in the first two columns of figure 7 . Here, the regions colored in purple (green) correspond to regions that gained (lost) electron density in the composite system. Clearly, with both basis sets, the major changes in the electron density are near the adsorption site, with a clear gain in the electron density between the carbon atom of the $\mathrm{CO}$ and the $\mathrm{Zn}$ atom proximal to the $\mathrm{CO}$. This gain in the electron density suggests the formation of $\mathrm{Zn}-\mathrm{C}$ bond in the combined system.

In the last column of figure 7, we have shown the difference in the interaction between the adsorbate and the slab with a change in the basis by subtracting the $\rho$ [diff] values calculated at each basis set (i.e. $\rho^{\text {basis }}$ diff] $=\rho^{\mathrm{TZ}}$ [diff] $-\rho^{\mathrm{DZ}}[$ diff]). Here, the regions colored in purple (green) correspond to regions that have larger electron density with the TZ basis (DZ basis). Clearly, for the $\mathrm{DZ}$ basis (colored in green), there is a larger delocalization of the electron density between $\mathrm{Zn}$ and $\mathrm{C}$ and can be related to the higher adsorption energy of $\mathrm{CO}$ in the $\mathrm{DZ}$ basis. On the other hand, for the TZ basis (colored in purple), there is a larger electron density localized at the $\mathrm{C}$ and $\mathrm{O}$ atoms (of $\mathrm{CO}$ ) suggesting a relatively weaker $\mathrm{Zn}-\mathrm{C}$ bond. Thus, the differences in the collision results with a change in the basis can be closely related to the differences in the interaction between an adsorbate molecule and the slab with a change in basis. We have also generated similar plots for the $\mathrm{CO}_{2}$ molecule that are given in the figure S17. For the case of $\mathrm{CO}_{2}, \rho^{\text {basis }}$ [diff] is negligible, suggesting that its interaction with the $\mathrm{ZnO}$ slab is less sensitive to changes in the basis set. This similarity in the interaction between $\mathrm{CO}_{2}$ and $\mathrm{ZnO}$, irrespective of the employed basis sets, can also be seen in the adsorption energies (table 1 ).
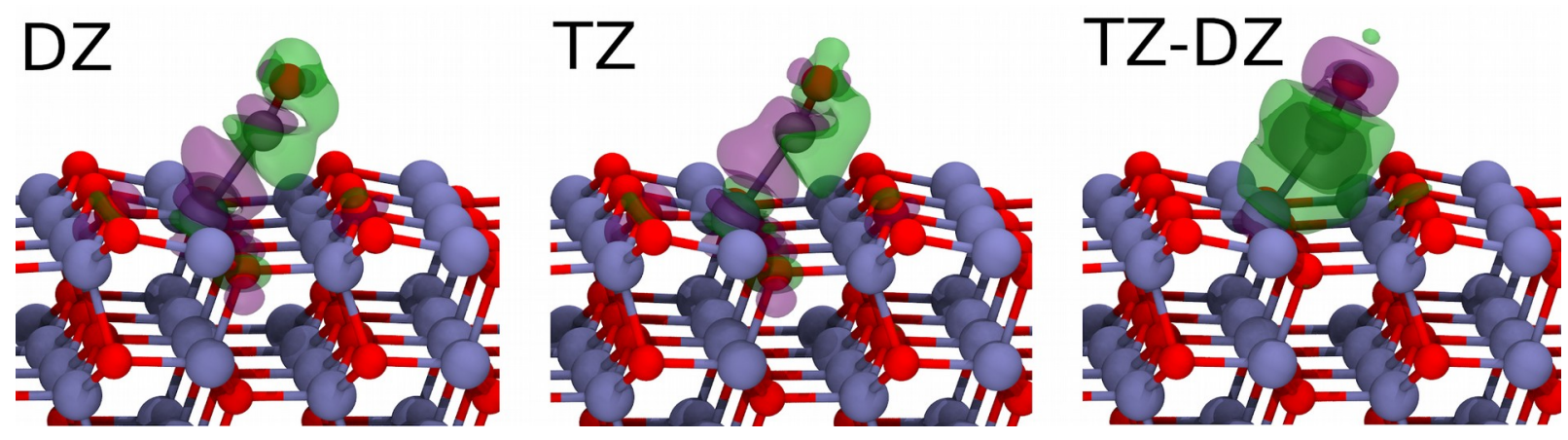
Figure 7: The first two columns shows electron density differences obtained for a single co molecule adsorbed on a ZnO slab with the DZVP (DZ) and TZV2P (TZ) basis-sets, respectively. Here, each of these columns is obtained by subtracting the total electron density of the entire system from its individual components (i.e. $\rho$ [diff] $=$ $\rho[\mathrm{ZnO}+\mathrm{mol}]-\rho[\mathrm{ZnO}]-\rho[\mathrm{mol}])$. The last column shows the difference in the densities between the first two columns (i.e. $\rho^{\text {basis }}\left[\right.$ diff] $=\rho^{\text {TZ }}$ diff] $-\rho^{\text {DZ }}$ [diff]). We have used an iso-value of 0.001 e $\AA^{-3}$ for the first two columns and 0.0003 e $\AA^{-}$

${ }^{3}$ for the last column.

\section{Results with other software packages}

To verify the above findings, we have repeated the adsorption energy calculations with the FHI-aims software package with two different basis-sets (tier-1 and tier-2, with tier-2 being the higher quality basis-set) and the results are reported in table 2. Once again, among all the adsorbate molecules, $\mathrm{CO}$ is the outlier with a difference of $\sim 0.1 \mathrm{eV}$ in the adsorption energy with a change in the basis, proving the robustness of our findings. We have also plotted (not shown here) $\rho^{\text {basis }}\left[\right.$ diff] for $\mathrm{CO}$ and $\mathrm{CO}_{2}$ using the $\mathrm{FHI}$-aims package and found them to be similar to the ones in figure 7 . Thus, we confirmed that the basis set does have an impact on the interaction between the adsorbate and surface, and this impact is immense for the case of $\mathrm{CO}$ with $\mathrm{ZnO}$. We have also verified these adsorption energy results with VASP (see Table S12). Finally, we have also demonstrated that the above findings are robust against the employed exchangecorrelation functional as well as the inclusion/omission of dispersion interactions (see Supporting Information).

Table 2: Adsorption energies of the molecules calculated using FHI-aims with two different basis sets and differences in the adsorption energies between basis sets.

\begin{tabular}{|c|c|c|c|}
\hline System & $\begin{array}{c}\text { Adsorption } \\
\text { energy } \\
\text { with the Tier-1 } \\
\text { basis, } \\
\mathrm{E}_{\text {Tier1 }}(\mathrm{eV})\end{array}$ & $\begin{array}{c}\text { Adsorption } \\
\text { energy } \\
\text { with the Tier-2 } \\
\text { basis, } \\
\mathrm{E}_{\text {Tier2 }}(\mathrm{eV})\end{array}$ & $\begin{array}{c}\text { Difference in } \\
\text { Adsorption energy, } \\
\left(\mathrm{E}_{\text {Tier2 }}-\mathrm{E}_{\text {Tier1 }}\right),(\mathrm{eV})\end{array}$ \\
\hline $\mathrm{ZnO} \mathrm{Slab}+\mathrm{CO}$ & -0.489 & -0.398 & 0.091 \\
\hline $\mathrm{ZnO} \mathrm{Slab}+\mathrm{CO}_{2}$ & -0.196 & -0.172 & 0.023 \\
\hline $\mathrm{ZnO} \mathrm{Slab}+\mathrm{H}_{2}$ & -0.095 & -0.096 & -0.001 \\
\hline $\mathrm{ZnO} \mathrm{Slab}+\mathrm{H}_{2} \mathrm{O}$ & -1.055 & -0.999 & 0.056 \\
\hline
\end{tabular}

\section{Conclusions and outlook}

Using ab initio molecular dynamics, we have examined a collision between the reactants of a water-gas shift reaction (i.e., $\mathrm{CO}$ and $\mathrm{H}_{2} \mathrm{O}$ ) with a $\mathrm{ZnO}$ surface and have shown how the resulting mechanism and dynamics are affected by the quality of the employed basis set. For both the low- and high-energy collisions, we have shown significant differences in the outcomes of a BOMD simulation by merely changing the basis set quality. While the differences are 
noticeable with the low-energy collision, they are quite substantial in the case of the high-energy collision. By analyzing the energetics of these collision reactions, we have shown that the KE gain by the adsorbate molecule from the colliding molecule is the same irrespective of the employed basis set. Using this result, we have determined the source of these differences to arise from the interaction between the surface and the adsorbate. Furthermore, through our binding energy calculations and electron density difference maps we have clearly shown that the interaction between the adsorbate and the substrate relies heavily on quality of the employed basis set. These results have significant ramifications for understanding catalytic dynamics since merely changing the quality of the basis for $\mathrm{CO}$ translates to more than an order of magnitude $(\sim 0.4 \mathrm{eV})$ energy difference compared to room-temperature $(\sim 0.026 \mathrm{eV})$. We have shown that these surprising results are robust by obtaining very similar trends with other software packages. Due to this sensitive dependence on basis sets, previous studies that have employed the commonly-used double zeta basis sets may need to be re-examined (especially for CO-based reactions).

Moving forward, we suggest that conducting a single-point binding energy calculation with different quality basis sets be first carried out as a diagnostic test to ensure that a suitable/reliable basis set is chosen prior to carrying out the computationally-demanding BOMD collision reactions. Specifically, if the resulting binding energies obtained with two different quality basis sets are similar, then the resulting BOMD dynamics can still reliably (and efficiently) use the lower-quality basis set. However, if the computed single-point binding energies from two different quality basis sets are dissimilar, extreme care should be taken since the resulting BOMD simulations will exhibit qualitatively different dynamics with these basis sets. Finally, although the conclusions drawn in this study pertain to basis set effects from BOMD simulations (where only the ground state PES is involved), we believe that these effects are quite general and will be applicable even to non-adiabatic molecular dynamics simulations, which is the focus of a future study.

\section{Acknowledgements}

The authors acknowledge the support of the U.S. Army Research Office under grant number W911NF-17-1-0340. This research used resources of the National Energy Research Scientific

Computing Center (NERSC), a U.S. Department of Energy Office of Science User Facility operated under Contract No. DE-AC02-05CH11231.

\section{Supporting Information}

The supporting information contains complete computational details, various convergence tests, BOMD calculations with different exchange-correlation functionals and impact positions, NBO and Mulliken analyses, additional discussions, and supporting videos, figures, and tables. 
The Supporting Information is available free of charge on the ACS Publications website at DOI:

\section{References}

(1) Bonn, M.; Funk, S.; Hess, C.; Denzler, D. N.; Stampfl, C.; Scheffler, M.; Wolf, M.; Ertl, G. Phonon- versus Electron-Mediated Desorption and Oxidation of $\mathrm{CO}$ on Ru(0001). Science 1999, 285, 1042-1045.

(2) Park, J. Y.; Baker, L. R.: Somorjai, G. A. Role of Hot Electrons and Metal-Oxide Interfaces in Surface Chemistry and Catalytic Reactions. Chem. Rev. 2015, 115, 2781-2817.

(3) Gavnholt, J.; Rubio, A.; Olsen, T.; Thygesen, K. S.; Schiøtz, J. Hot-Electron-Assisted Femtochemistry at Surfaces: A Time-Dependent Density Functional Theory Approach. Phys. Rev. B: Condens. Matter Mater. Phys. 2009, 79. https://doi.org/10.1103/physrevb.79.195405.

(4) Wintterlin, J.; Schuster, R.; Ertl, G. Existence of a "Hot" Atom Mechanism for the Dissociation ofO2on Pt(111). Phys. Rev. Lett. 1996, 77, 123-126.

(5) Blanco-Rey, M.; Juaristi, J. I.; Díez Muiño, R.; Busnengo, H. F.; Kroes, G. J.; Alducin, M. Electronic Friction Dominates Hydrogen Hot-Atom Relaxation on Pd(100). Phys. Rev. Lett. 2014, 112, 103203.

(6) Pétuya, R.: Larrégaray, P.: Crespos, C.: Aurel, P.: Busnengo, H. F.: Martínez, A. E. Scattering of Atomic Hydrogen Off a H-Covered W(110) Surface: Hot-Atom versus EleyRideal Abstraction Dynamics. J. Phys. Chem. C 2015, 119, 3171-3179.

(7) Brongersma, M. L.; Halas, N. J.; Nordlander, P. Plasmon-Induced Hot Carrier Science and Technology. Nat. Nanotechnol. 2015, 10, 25-34.

(8) Liu, G.; Li, P.; Zhao, G.; Wang, X.; Kong, J.; Liu, H.; Zhang, H.; Chang, K.; Meng, X.; Kako, T.; et al. Promoting Active Species Generation by Plasmon-Induced Hot-Electron Excitation for Efficient Electrocatalytic Oxygen Evolution. J. Am. Chem. Soc. 2016, 138, 9128-9136.

(9) Sakamoto, H.; Ohara, T.; Yasumoto, N.; Shiraishi, Y.; Ichikawa, S.; Tanaka, S.; Hirai, T. HotElectron-Induced Highly Efficient O2 Activation by Pt Nanoparticles Supported on Ta2O5 Driven by Visible Light. J. Am. Chem. Soc. 2015, 137, 9324-9332.

(10) Avanesian, T.; Christopher, P. Adsorbate Specificity in Hot Electron Driven Photochemistry on Catalytic Metal Surfaces. J. Phys. Chem. C 2014, 118, 28017-28031.

(11) Park, J. Y.; Kim, S. M.; Lee, H.; Nedrygailov, I. I. Hot-Electron-Mediated Surface Chemistry: Toward Electronic Control of Catalytic Activity. Acc. Chem. Res. 2015, 48, 2475 2483.

(12) Bork, N.; Loukonen, V.; Vehkamäki, H. Reactions and Reaction Rate of Atmospheric $\mathrm{SO} 2$ and $\mathrm{O} 3-(\mathrm{H} 2 \mathrm{O}) n$ Collisions via Molecular Dynamics Simulations. J. Phys. Chem. A 2013, 117, 3143-3148.

(13) Tsona, N. T.; Bork, N.; Loukonen, V.; Vehkamäki, H. A Closure Study of the Reaction between Sulfur Dioxide and the Sulfate Radical Ion from First-Principles Molecular Dynamics Simulations. J. Phys. Chem. A 2016, 120, 1046-1050.

(14) Olsen, R. A.; Kroes, G. J.; Henkelman, G.; Arnaldsson, A.; Jónsson, H. Comparison of Methods for Finding Saddle Points without Knowledge of the Final States. J. Chem. Phys. 2004, 121, 9776-9792.

(15) Henkelman, G.; Jóhannesson, G.; Jónsson, H. Methods for Finding Saddle Points and Minimum Energy Paths. In Progress in Theoretical Chemistry and Physics: pp 269-302.

(16) Zhou, X.; Zhang, L.; Jiang, B. Hot-Atom-Mediated Dynamical Displacement of CO Adsorbed on $\mathrm{Cu}(111)$ by Incident H Atoms: An Ab Initio Molecular Dynamics Study. J. Phys. Chem. C 2018, 122, 15485-15493.

(17) Wang, Z.-T.; Wang, Y.-G.; Mu, R.; Yoon, Y.; Dahal, A.; Schenter, G. K.; Glezakou, V.-A.; 
Rousseau, R.; Lyubinetsky, I.; Dohnálek, Z. Probing Equilibrium of Molecular and

Deprotonated Water on TiO2(110). Proceedings of the National Academy of Sciences

2017, 114, 1801-1805.

(18) Lin, W.; Stocker, K. M.; Schatz, G. C. Mechanisms of Hydrogen-Assisted CO2 Reduction on Nickel. J. Am. Chem. Soc. 2017, 139, 4663-4666.

(19) Ashwell, A. P.; Lin, W.; Hofman, M. S.; Yang, Y.; Ratner, M. A.; Koel, B. E.; Schatz, G. C. Hydrogenation of $\mathrm{CO}$ to Methanol on $\mathrm{Ni}(110)$ through Subsurface Hydrogen. J. Am. Chem. Soc. 2017, 139, 17582-17589.

(20) Lin, W.; Schatz, G. C. Mechanisms of Formaldehyde and C2 Formation from Methylene Reacting with CO2 Adsorbed on Ni(110). J. Phys. Chem. C 2018, 122, 13827-13833.

(21) Groß, A. Ab Initio Molecular Dynamics Simulations of the $02 / \mathrm{Pt}\left(\begin{array}{lll}1 & 1 & 1\end{array}\right)$ Interaction. Catal. Today 2016, 260,60-65.

(22) Zhou, X.; Kolb, B.; Luo, X.; Guo, H.; Jiang, B. Ab Initio Molecular Dynamics Study of Dissociative Chemisorption and Scattering of CO2 on Ni(100): Reactivity, Energy Transfer, Steering Dynamics, and Lattice Effects. J. Phys. Chem. C 2017, 121, 5594-5602.

(23) Li, L.; Zeng, X. C. Direct Simulation Evidence of Generation of Oxygen Vacancies at the Golden Cage Au16 and TiO2 (110) Interface for CO Oxidation. J. Am. Chem. Soc. 2014, 136, 15857-15860.

(24) Li, L.; Li, H.; Zeng. X. C. Structure Transition of Au18 from Pyramidal to a Hollow-Cage during Soft-Landing onto a TiO2(110) Surface. Chem. Commun. 2015, 51, 9535-9538.

(25) Hirshberg, B.; Benny Gerber, R. Formation of Carbonic Acid in Impact of CO2 on Ice and Water. J. Phys. Chem. Lett. 2016, 7, 2905-2909.

(26) VandeVondele, J.; Krack, M.; Mohamed, F.; Parrinello, M.; Chassaing, T.; Hutter, J. Quickstep: Fast and Accurate Density Functional Calculations Using a Mixed Gaussian and Plane Waves Approach. Comput. Phys. Commun. 2005, 167, 103-128.

(27) Blum, V.; Gehrke, R.; Hanke, F.; Havu, P.; Havu, V.; Ren, X.; Reuter, K.; Scheffler, M. Ab Initio Molecular Simulations with Numeric Atom-Centered Orbitals. Comput. Phys. Commun. 2009, 180, 2175-2196.

(28) Kresse, G.; Furthmüller, J. Efficiency of Ab-Initio Total Energy Calculations for Metals and Semiconductors Using a Plane-Wave Basis Set. Comput. Mater. Sci. 1996, 6, 15-50.

(29) Zhao, Y.; Truhlar, D. G. Density Functional Calculations of E2 and SN2 Reactions: Effects of the Choice of Density Functional, Basis Set, and Self-Consistent Iterations. J. Chem. Theory Comput. 2010, 6, 1104-1108.

(30) Narendrapurapu, B. S.: Richardson, N. A.; Copan, A. V.; Estep, M. L.: Yang. Z.: Schaefer, H. F. Investigating the Effects of Basis Set on Metal-Metal and Metal-Ligand Bond Distances in Stable Transition Metal Carbonyls: Performance of Correlation Consistent Basis Sets with 35 Density Functionals. J. Chem. Theory Comput. 2013, 9, 2930-2938.

(31) Gavin Williams, T.; Wilson, A. K. Importance of the Quality of Metal and Ligand Basis Sets in Transition Metal Species. J. Chem. Phys. 2008, 129, 054108.

(32) $\quad \underline{X u}, \mathrm{X}$; Truhlar, D. G. Accuracy of Effective Core Potentials and Basis Sets for Density Functional Calculations, Including Relativistic Effects, As Illustrated by Calculations on

Arsenic Compounds. J. Chem. Theory Comput. 2011, 7, 2766-2779.

(33) Steele, R. P. Multiple-Timestep Ab Initio Molecular Dynamics Using an Atomic Basis Set Partitioning. J. Phys. Chem. A 2015, 119, 12119-12130.

(34) Li, J.; Iyengar, S. S. Ab Initio Molecular Dynamics Using Recursive, Spatially Separated, Overlapping Model Subsystems Mixed within an ONIOM-Based Fragmentation Energy

Extrapolation Technique. J. Chem. Theory Comput. 2015, 11, 3978-3991.

(35) Haycraft, C.; Li, J.; Iyengar, S. S. Efficient, "On-the-Fly", Born-Oppenheimer and CarParrinello-Type Dynamics with Coupled Cluster Accuracy through Fragment Based Electronic Structure. J. Chem. Theory Comput. 2017, 13, 1887-1901. 
(36) Steele, R. P.; DiStasio, R. A., Jr; Head-Gordon, M. Non-Covalent Interactions with DualBasis Methods: Pairings for Augmented Basis Sets. J. Chem. Theory Comput. 2009, 5, 1560-1572.

(37) Steele, R. P.; Head-Gordon, M. Dual-Basis Self-Consistent Field Methods: 6-31G* Calculations with a Minimal 6-4G Primary Basis. Molecular Physics. 2007, pp 2455-2473. https://doi.org/10.1080/00268970701519754.

(38) Steele, R. P.; Head-Gordon, M.; Tully, J. C. Ab Initio Molecular Dynamics with Dual Basis Set Methods. J. Phys. Chem. A 2010, 114, 11853-11860.

(39) Ricard, T. C.; Iyengar, S. S. Efficiently Capturing Weak Interactions in Ab Initio Molecular Dynamics with on-the-Fly Basis Set Extrapolation. J. Chem. Theory Comput. 2018, 14. 5535-5552.

(40) Rodriguez, J. A.; Liu, P.; Hrbek, J.; Evans, J.; Pérez, M. Water Gas Shift Reaction on Cu and $\mathrm{Au}$ Nanoparticles Supported on $\mathrm{CeO} 2(111)$ and $\mathrm{ZnO}(000)$ : Intrinsic Activity and Importance of Support Interactions. Angew. Chem. Int. Ed Engl. 2007, 119, 1351-1354.

(41) Tabatabaei, J.; Sakakini, B. H.; Waugh, K. C. On the Mechanism of Methanol Synthesis and the Water-Gas Shift Reaction on ZnO. Catal. Letters 2006, 110, 77-84.

(42) Nakatsuji, H.; Yoshimoto, M.; Umemura, Y.; Takagi, S.; Hada, M. Theoretical Study of the Chemisorption and Surface Reaction of $\mathrm{HCOOH}$ on a $\mathrm{ZnO}(1010)$ Surface. J. Phys. Chem. 1996. 100, 694-700.

(43) Wang, D.; Ma, L.; Jiang, C. J.; Trimm, D. L.; Wainwright, M. S.; Kim, D. H. The Effect of Zinc Oxide in Raney Copper Catalysts on Methanol Synthesis, Water Gas Shift, and Methanol Steam Reforming Reaction. In Studies in Surface Science and Catalysis; 1996; pp 1379-1387.

(44) Álvarez Galván, C.; Galván, C. Á.; Schumann, J.; Behrens, M.; Fierro, J. L. G.; Schlögl, R.; Frei, E. Reverse Water-Gas Shift Reaction at the Cu/ZnO Interface: Influence of the Cu/Zn Ratio on Structure-Activity Correlations. Appl. Catal. B 2016, 195, 104-111.

(45) Perdew, J. P.; Burke, K.; Ernzerhof, M. Generalized Gradient Approximation Made Simple [Phys. Rev. Lett. 77, 3865 (1996)]. Phys. Rev. Lett. 1997, 78, 1396-1396.

(46) VandeVondele, J.; Hutter, J. Gaussian Basis Sets for Accurate Calculations on Molecular Systems in Gas and Condensed Phases. J. Chem. Phys. 2007, 127, 114105.

(47) Goedecker, S.; Teter, M.; Hutter, J. Separable Dual-Space Gaussian Pseudopotentials. Phys. Rev. B: Condens. Matter Mater. Phys. 1996, 54, 1703-1710.

(48) Krack, M. Pseudopotentials for $\mathrm{H}$ to Kr Optimized for Gradient-Corrected ExchangeCorrelation Functionals. Theor. Chem. Acc. 2005, 114, 145-152.

(49) Shi, H.; Yuan, H.; Ruan, S.; Wang, W.; Li, Z.; Li, Z.; Shao, X. Adsorption and Diffusion of $\mathbb{C O}$ on Clean and CO2-Precovered ZnO(1010). J. Phys. Chem. C 2018, 122, 8919-8924.

(50) Grimme, S.; Ehrlich, S.; Goerigk, L. Effect of the Damping Function in Dispersion Corrected Density Functional Theory. J. Comput. Chem. 2011, 32, 1456-1465.

(51) Meyer, B.; Marx, D. First-Principles Study of CO Adsorption on ZnO Surfaces. J. Phys. Condens. Matter 2003, 15, L89-L94. 
TOC graphic
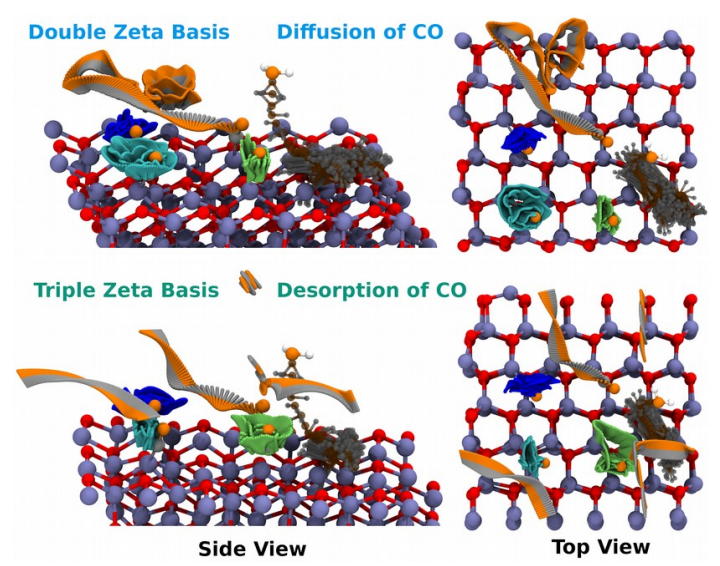